\title{
How can we use dendrimer-templated ultrasmall and multifunctional nanoparticles in photothermal cancer therapy?
}

\author{
"Dendrimers have a well-defined size, shape, hollow interior \\ and homogenous surface functional groups, especially \\ poly(amidoamine) dendrimers, which possess a spherical \\ shape and ultrasmall sizes. It would be a desirable scaffold \\ for the fabrication of theranostic agents with renal clearable \\ characteristics."
}

First draft submitted: 10 October 2016; Accepted for publication: 21 October 2016; Published online: 15 November 2016

\section{Keywords: cancer therapy $\bullet$ dendrimer $\bullet$ multifunctional $\bullet$ photothermal therapy} - ultrasmall nanoparticles

Nanomaterial-mediated photothermal therapy (PTT) has been broadly studied for the treatment of cancer in the past decade. Nanomaterials with high near-infrared absorbance can convert near-infrared light into heat to enhance the local temperature of tumors and therefore cause the irreversible damage of cancer cells and finally the elimination of tumors. The previous research interests focus on the development of new compositions of nanomaterials with high photothermal conversion efficiency, and also keen on demonstrating the efficacy of PTT in ablation of different tumors [1]. To date, there have been a variety of photothermal nanomaterials including metal nanoparticles, carbon-based nanomaterials, semiconductor nanoparticles and polymer nanoparticles [2]. The gold nanoshells have even been approved for the treatment of two human cancers in clinical trials [3]. While PTT is infinitely approaching the clinical translation, there have been tremendous studies to invent new ways of enhancing therapeutic efficacy and reducing the toxicity of photothermal nanomaterials.

\section{Dendrimer-templated synthesis of ultrasmall photothermal nanoparticles}

Unlike the conventional imaging agents and therapeutics, the photothermal nanomaterials possess a relatively larger size, and are always retain in the body for a long period or even permanently after the treatments [4]. Although the studies in literatures always suggest that the photothermal nanomaterials are highly biocompatible, these demonstrations are not systematic and comprehensive. A long-term retention of nanomaterials in the body might cause the foreign body reaction and other unexpected adverse effects [5]. Some of the nanomaterials contain heavy metals which retain in the body for a long time and will also interfere with the subsequent diagnostic imaging [6]. Additionally, the physiological conditions of individual patients are dissimilar, and thus their responses and tolerances to these therapeutic nanoparticles would also be different.

A desirable way to minimize the potential toxicity of nanomaterials is to clear them out of the body post-treatment. Renal filtra-

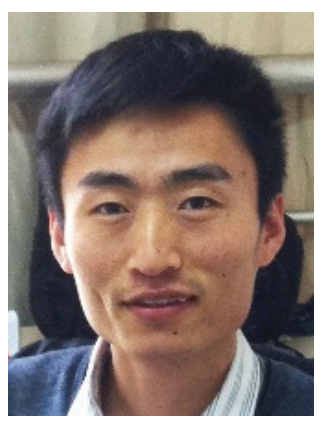

Qiang Zhang

Shanghai Key Laboratory of Regulatory Biology, School of Life Sciences, East China Normal University, Shanghai, 200241, PR China

Zhengjie Zhou

Shanghai Key Laboratory of Regulatory Biology, School of Life Sciences, East China Normal University, Shanghai, 200241, PR China

\section{Yitong Wang}

Shanghai Key Laboratory of Regulatory Biology, School of Life Sciences, East China Normal University, Shanghai, 200241, PR China

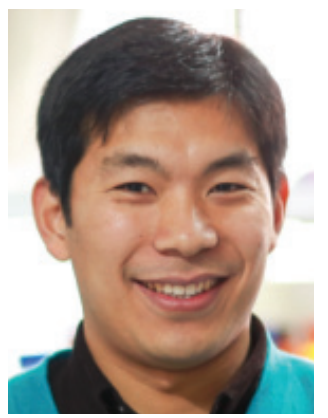

Yiyun Cheng

Author for correspondence: Shanghai Key Laboratory of Regulatory Biology, School of Life Sciences, East China Normal University, Shanghai, 200241, PR China

yycheng@mail.ustc.edu.cn

Future $\%$ Medicine $\%$ 
tion represents an optimal pathway for the clearance of nanomaterials as it is minimally involved in the intracellular catabolism compared with hepatic secretion [7], which reduces the retention and cytotoxicity of nanomaterials in the body. The previous investigations suggest that filtration of nanomaterials through kidney is highly dependent on the material characteristics such as size, shape, surface modification and charge [4]. In particular, the hydrodynamic size of nanomaterials is basically required to be less than $10 \mathrm{~nm}$ [8]. However, the developed photothermal nanomaterials are mostly larger than $10 \mathrm{~nm}$, which therefore are not able to be cleared out of the body via renal filtration. Dendrimers have a well-defined size, shape, hollow interior and homogenous surface functional groups, especially poly(amidoamine) (PAMAM) dendrimers, which possess a spherical shape and ultrasmall sizes. It would be a desirable scaffold for the fabrication of theranostic agents with renal clearable characteristics [9]. The previous studies have demonstrated that PAMAM dendrimers of less than sixth generation or smaller than $5.4 \mathrm{~nm}$ in diameter are clearable for renal filtration [10].

Therefore, we employed the amine-terminated generation fifth $\left(\mathrm{G} 5-\mathrm{NH}_{2}\right)$ PAMAM dendrimers as a template to synthesize different photothermal nanoparticles with ultrasmall size [11]. We found that the platinum, palladium and copper sulfide nanoparticles, all represented an excellent photothermal effect and demonstrated that they could be cleared out of the body via renal filtration. Our study paves a facile and robust route to fabricate photothermal nanoparticles with renal clearance characteristics. In the further study, PAMAM dendrimers that are smaller than sixth generation can all be used to synthesize diverse ultrasmall photothermal nanoparticles, and even other kinds of dendrimers can also be tested for the synthesis of ultrasmall photothermal nanoparticles. New compositions of materials can further be explored to enrich the variety of ultrasmall photothermal agents and to improve the photothermal conversion efficiency. The high density of surface functional groups on dendrimers provides a desirable opportunity to investigate the influence of surface modification and charge over renal clearance. Based on this study, we are able to find out an optimal photothermal nanoparticle with all the advanced properties including excellent biocompatibility, high photothermal effect and optimal renal clearance characteristics.

\section{Multi-functionalization of ultrasmall photothermal nanoparticles}

Nanoparticle-based therapeutics have been developed with additional capabilities like targeting, imaging and new therapeutics, which can also significantly improve the therapeutic efficacy and reduce the systematic toxicity of the therapeutic agents [7]. For instance, targeting therapeutic agents to the pathologic lesions and/or the combined therapeutics must enhance the therapeutic efficacy, and thus allows the administration of minimal therapeutic agents to reduce the potential toxicity. However, additional functionality means complicated synthetic steps and additional costs [12]. Additional synthetic steps also bring huge difficulty to keep different components consistent in the formulation [13]. To modify functional groups on ultrasmall photothermal nanoparticles will become more difficult due to their ultrasmall sizes and limited structure variations. Nevertheless, it is not a problem in our case. PAMAM dendrimers possess high density of functional groups such as amine, hydroxyl and carboxyl groups on their surfaces. For instance, there are 128 amine groups on a G5- $\mathrm{NH}_{2}$ PAMAM dendrimer. The well-developed strategy for dendrimers allows accurately decorating dissimilar moieties on their surfaces with designed numbers and placements. In our case, we first modified 100 acetyl groups on the surface of G5- $\mathrm{NH}_{2}$ PAMAM dendrimers to improve their biocompatibility, and then we modified three RGD peptides and one fluorescent probe cyanine 5.5 on the surface of dendrimers [6]. The amounts of all these moieties modified on dendrimers could be well controlled in the synthesis, and further could be accurately determined using nuclear magnetic resonance technique, which guarantees the consistency of components in the formulation, and further assures the reproducible pharmacokinetic behavior of the therapeutic agents in the body.

Dendrimers can be further decorated with multiple imaging modalities like fluorescent probes and contrast agents for magnetic resonance imaging or computed tomography to complement the intrinsic limitations of individual components. The interiors of dendrimers are not restricted to encapsulate photothermal nanoparticles, but can also be used to load other therapeutic agents like chemical drugs. Moreover, drug molecules can also be loaded on the surface of dendrimers. Therefore, other therapeutics such as chemotherapy and photodynamic therapy can be incorporated in dendrimertemplated photothermal nanoparticles to conduct a combined therapy.

There is a paradox for the ultrasmall photothermal nanoparticles. The ultrasmall nanoparticles can be quickly cleared out of the body via renal secretion to reduce their potential toxicity. On the other hand, it requires a relative long-term retention of the nanoparticles in tumors to conduct repeated PTTs. In our previous case, we had no choice except to administrate multiple doses of ultrasmall photothermal nanoparticles for tumor ablation [11], which not only caused 
discomfort to patients but also might increase dosecumulating side effects. To deal with this problem, a temperature-responsive polymer such as PNIPAM can be modified on the dendrime-templated ultrasmall photothermal nanoparticles, which can undergo a phase transition from hydrophilic to hydrophobic state in tumor and thus form large aggregates to prolong their retention. PNIPAM can also be modified on the surface of ultrasmall photothermal nanoparticles via biodegradable bond to faciliate their degradation and clearance via renal filtration.

\section{Outlook}

Looking forward, there are great consequences and opportunities for researchers to look for advanced ultrasmall and multifunctional photothermal agents based on dendrimers. By changing the nanoparticle compositions and varying the surface modifications, we can obtain the ultrasmall photothermal nanoparticles with both highly photothermal conversion efficiency

\section{References}

1 Zhang Z, Wang J, Chen C. Near-infrared light-mediated nanoplatforms for cancer thermo-chemotherapy and optical imaging. Adv. Mater. 25, 3869-3880 (2013).

2 Cheng L, Wang C, Feng L, Yang K, Liu Z. Functional nanomaterials for phototherapies of cancer. Chem. Rev. 114, 10869-10939 (2014).

3 Melamed JR, Edelstein RS, Day ES. Elucidating the fundamental mechanisms of cell death triggered by photothermal therapy. ACS Nano 9, 6-11 (2015).

4 Longmire M, Choyke PL, Kobayashi H. Clearance properties of nano-sized particles and molecules as imaging agents: considerations and caveats. Nanomedicine 3, 703-717 (2008).

5 Tang S, Chen M, Zheng N. Sub-10-nm Pd nanosheets with renal clearance for efficient near-infrared photothermal cancer therapy. Small 10, 3139-3144 (2014).

6 Dong W, Li Y, Niu D et al. Facile synthesis of monodisperse superparamagnetic $\mathrm{Fe}_{3} \mathrm{O}_{4}$ core@hybrid@Au shell nanocomposite for bimodal imaging and photothermal therapy. Adv. Mater. 23, 5392-5397 (2011).

7 Choi HS, Liu W, Misra P et al. Renal clearance of quantum dots. Nat. Biotechnol. 25, 1165-1170 (2007). and excellent renal clearance characteristics. Furthermore, additional functionalities like targeting moieties, imaging modalities and therapeutics are able to be incorporated in the ultrasmall photothermal nanoparticles to further enhance the therapeutic efficacy and reduce toxicity. We believe that the optimal ultrasmall and multifunctional photothermal nanoparticles would significantly contribute to PTT of cancer.

\section{Financial \& competing interests disclosure}

This work was supported by the Basic Research Program of Science and Technology Commission of Shanghai Municipality (grant no. 14JC1491100), and the Shanghai Pujiang Program (grant no. 14PJD016). The authors have no other relevant affiliations or financial involvement with any organization or entity with a financial interest in or financial conflict with the subject matter or materials discussed in the manuscript apart from those disclosed.

No writing assistance was utilized in the production of this manuscript.
8 Liu J, Yu M, Zhou C, Zheng J. Renal clearable inorganic nanoparticles: a new frontier of bionanotechnology. Mater. Today 16, 477-486 (2013).

9 Yang J, Zhang Q, Chang H, Cheng Y. Surface-engineered dendrimers in gene delivery. Chem. Rev. 115, 5274-5300 (2015).

10 Kobayashi H, Brechbiel MW. Nano-sized MRI contrast agents with dendrimer cores. Adv. Drug Deliv. Rev. 57, 2271-2286 (2005).

11 Zhou Z, Wang Y, Yan Y, Zhang Q, Cheng Y. Dendrimertemplated ultrasmall and multifunctional photothermal agents for efficient tumor ablation. ACS Nano 10, 4863-4872 (2016).

12 Cheng Z, Zaki AA, Hui JZ, Muzykantov VR, Tsourkas A. Multifunctional nanoparticles: cost versus benefit of adding targeting and imaging capabilities. Science 338, 903-910 (2012).

13 Song XR, Wang X, Yu SX et al. Co9 Se8 nanoplates as a new theranostic platform for photoacoustic/ magnetic resonance dual-modal-imaging-guided chemophotothermal combination therapy. Adv. Mater. 27, 3285-3291 (2015). 